\title{
Numerical simulation of two-dimensional laminar unsteady flow past a right trapezoidal cylinder at low Reynolds number: study of sharpening angle, time step, grid independence and domain size
}

\author{
Sodsai Lamtharn ${ }^{1, *}$, Monsak Pimsarn ${ }^{1}$ \\ ${ }^{1}$ Mechanical Engineering Department, Faculty of Engineering, King Mongkut's Institute of Technology Ladkrabang, Bangkok, \\ Thailand
}

\begin{abstract}
Numerical simulation of two-dimensional laminar unsteady flow past a right trapezoidal cylinder at low Reynolds number $(R e=100)$, zero of the flow approaching angle and sharpening angle of the right trapezoidal of $22.5^{\circ}$ with a side ratio $B / A=1$ are carried out to provide more applicable data for engineering design of barred tee in aspect of structural integrity. A finite volume method, non-uniform meshing with second-order implicit time discretization into eightnode quadratic quadrilateral finite elements is employed. An incompressible flow SIMPLEC code with constant fluid properties is used. The convective terms using a third-order QUICK scheme. The numerical simulation result is compared against the published results of flow past a square cylinder. The effect of sharpening angle on the response of the right trapezoidal cylinder is investigated. A special study of the effects of flow on significant factor for time step, grid independence, blockage ratio, domain size, upstream and downstream extents, size domain next to cylinder and size domain extent are performed systematically. The Strouhal number and RMS lift coefficients of fully saturated flow are calculated. The result shown that increasing of sharpening angle, the Strouhal number is negligible changed whilst the RMS lift coefficients significantly increased.
\end{abstract}

\section{Introduction}

In long piping or pipeline system normally used pigging for various internal activities such as separating fluids, cleaning and inspecting the pipeline. Pig is a spherically shaped device which can be propelled through a main pipeline by pressurized fluid flow from pig trap launcher through main line, branch line and together with all

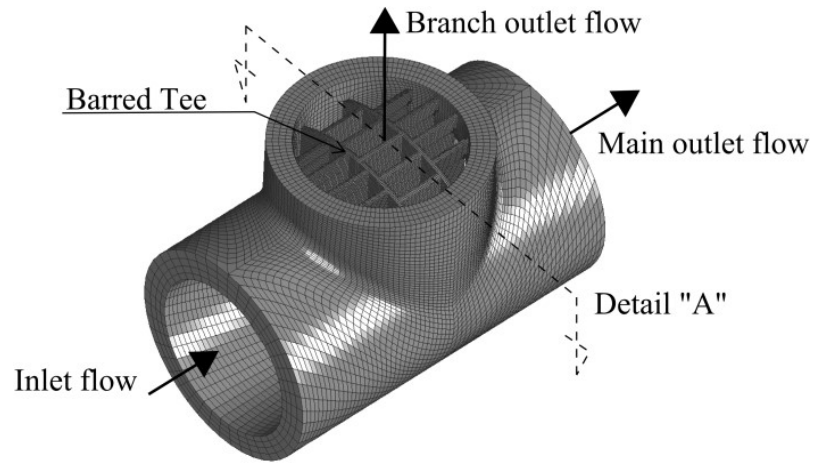

Fig. 1. Schematic of flow past barred tee associated piping, valves, pipe supports and instruments to pig trap receiver. The main line is major portion of a pipeline, between pig traps which compose many branch connection line. When piping or pipeline system is liquid filled into system to operate, the flow past main line and flow into branch line and past barred tee. In order to prevent the passing of pigs/spheres through branch pipe as show on Fig. 1. and Fig. 2. the barred tees (guided bars) is provided, filled-welded attached cross inside branch at connection point. Typically barred tees be installed on all branches larger than $50 \%$ or $25 \%$ of the main pipeline diameter where unwrapped foam pigs or spheres are to be used.

The barred tees have to integrity against to fluids flow induced vibration. In engineering design point of view, we concern to the synchronize between natural frequency of barred tee with vortex shedding frequency of fluid flow as past barred tee, the synchronize vibration of them cause barred tee experienced severe vibration then fatigue, detached from wall pipe into main pipe line which can be resulted pig stuck, valve face crash or scraper flow in to other inline equipment such as flow control valve and pump impeller. More additional cause

Corresponding author: sodsai.1@hotmail.co.th 


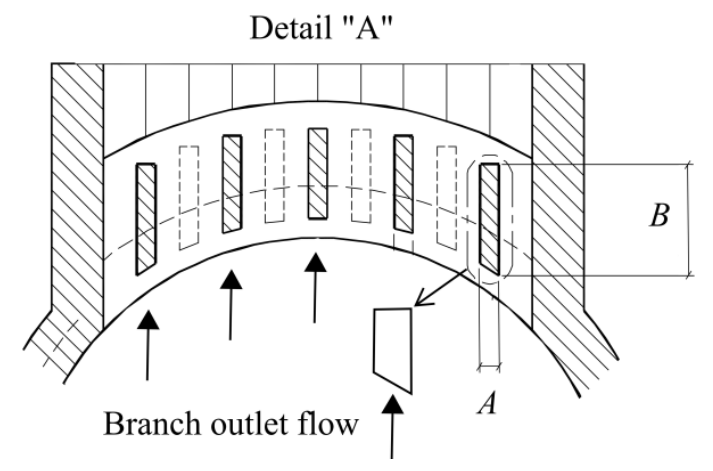

Fig. 2. Cross-section of barred tee

piping or pipeline stress exceed at branch connection. As shown on Fig. 2. the flow past a barred tee in which composed with several cross-section shape of a square, a rectangular and a right trapezoidal cylinder.

By numerical simulation, the enormous increase on the powerful processing of modern computers simulation has made it possible to identify and to study several mechanisms on flow past two dimensional cylindrical bluff body, many work used numerical simulation for flow past over cylindrical shape.

Sohankar et al. [1] carried out numerical simulations of two dimensional unsteady flow around a square cylinder at zero approaching angle $\alpha=0^{\circ}, R e=$ 100 to study the influence of time step $\Delta t$, size domain next to cylinder $\zeta$, grid size, extend of computation domain size $\Delta$, upstream extends $X_{u}$ and downstream extends $X_{d}$ of the calculation domain, side wall height, respectively. The method for numerical simulation is an incompressible SIMPLEC finite volume code, nonstaggered grid arrangement and Neumann outlet boundary condition. The convective terms are a thirdorder QUICK scheme and a second-order Van Leer scheme. Implicit time discretization and second-order Crank-Nicolson scheme is employed. For accurate calculation [1] finally used of $\Delta t=0.025$ and $\zeta=0.004$. The appropriate value of $X_{u}=11.1$ and $X_{d} \geq 25$ units.

Sohankar et al. [2] performed and provide a number of quantities same as [1] with rectangular cylinders at $\alpha=0^{\circ}-90^{\circ}$ with $R e \leq 200$, ratio of elongated side $B$ per short side $A=1-4$. The Strouhal number $S t$ and RMS lift coefficient $C l$ ' at $B / A=1$ are increase where $R e$ increase up to angle of approaching about $45^{\circ}$ then it decrease symmetrically.

Sohankar et al. [3] calculated and provide a number of quantities same as [1-2] a square cylinder at $\alpha$ $=0^{\circ}-45^{\circ}$ and $R e=45-200$ included studied the outlet of the computational domain for a convective Sommerfeld boundary condition (CBC) and a traditional Neumann condition (NBC). For CBC is suitable for flow around a square cylinder with non-zero angle of approaching and the $\mathrm{CBC}$ more effective in reducing the CPU time, reducing the upstream influence from the outlet and reducing the necessary downstream extent. Within $R e=$ 45-200, [3] recommended to use appropriate value $X_{d}>$ 15 units for $R e<100$ and $X_{d}>10$ units for $R e=100-200$ for $\mathrm{CBC}$ and the upstream influence of the outlet is effectively damped out on NBC at $X_{d}>25$ units approximately. The blockage ratio $\gamma$ reduced from $5 \%$ to
$2 \cdot 5 \%$ for $R e=100-200$ at $\alpha=0^{\circ}$ resulted in a small decrease in Strouhal number and RMS lift coefficient.

Ming et al. [4] investigated effect of flow approaching angle at $\alpha=0^{\circ}, 45^{\circ}$, and $22.5^{\circ}$ on the response of the square cylinder on vortex induced vibrations at $R e=100$ and a low mass ratio of 3 with the Navier-Stokes equations using the finite element method. Rectangular computational domain in the flow direction of a length of 60 units and a height in the crossflow direction of a length of 40 units are discretized into quadrilateral four-node linear finite elements. The center of the cylinder is distance of 20 units from the inlet boundary and equal distance to the up and down for lateral boundaries of 20 of the computational domain with $\gamma=2.5 \%$. Mesh dependence study is included in [4]. [4] also provide the Strouhal number and RMS lift coefficients at $\gamma=2.5 \%$ for $\alpha=0^{\circ}, 45^{\circ}$, and $22.5^{\circ}$.

Arnab et al. [5] performed numerical simulations of two-dimensional laminar flow past a triangular cylinder in which placed in free-stream at $10 \leq R e \leq 250$ by method a finite volume, second-order accurate in space and time, employing non-staggered arrangement of the variables with momentum interpolation for the pressure-velocity coupling. Result shown that vortex shedding phenomena of a triangular cylinder is same as to the square cylinder. Effect of grid refinement, $X_{u}$ and $X_{d}$ of the calculation domain are investigated and also study on the RMS lift coefficient and Strouhal number.

Mittal et al. [6] investigated effect of the blockage ratio on numerical simulation for flow past circular cylinder at $R e \leq 150$ with a stabilized space-time finite element formulation, $\gamma=1 \%$ and $5 \%$. From literature as mention above and in the past, the most applicable engineering design data for flow past over circular, squre cylinder, rectangular, trangle and isosceles trapezoidal are emphasis intense investigated but more absence applicable engineering design data for right trapezoidal cylindrical cross section in which to used in barred tee engineering design.

In this study, numerical simulations are carried out to investigate Strouhal number, RMS lift coefficient, of a right trapezoidal cylinder in unsteady flow to provide further more applicable data for engineering design of barred tee in aspect of structural integrity. The effects of time step, grid independence, sharpening angle $\beta$, blockage ratio, domain size, upstream and downstream extents, size domain next to trapezoidal cylinder are studied systematically. Fig. 3. show the schematic of the computational geometry of flow in this study and the definition of $\alpha, \beta, B, A, X_{u}, X_{d}$, vertical distance between the lower and upper walls of computational domain $H$ and projected width of the cylinder in the streamwise direction $d$. The streamwise and tranverse direction is placed on the $D$ and $L$ axis, respectively. The center of cylinder is on origin. The constant flow inlet velocity $U_{\infty}$ approached into side $A$ and $B$ at some degree of the $\alpha$ as depicted in Fig. 3. The Reynolds number is defined as $R e=U_{\infty} d / v$, where $d=A \cos (\alpha+\beta) / \cos \beta+B \sin \alpha, 0^{\circ} \leq \alpha \leq$ $(90-\beta)^{\circ}$ and $d=B \sin \alpha,(90-\beta)^{\circ}<\alpha \leq 90^{\circ}$ and $0^{\circ} \leq \beta \leq 60^{\circ}$ and $v$ is kinematic viscosity. All length dimensional are scaled to nondimensionless with $d$ included Strouhal number $S t=f_{S} d / U_{\infty}$, where $f_{S}$ is the shedding frequency. 
The confined boundary parameter which is blockage ratio is defined as $\gamma=d / H$, where velocities scaled with $U_{\infty}$. The origin of force coordinates is placed at the center of right trapezoidal cylinder geometry with drag and lift force positive in the $D$ and $L$-direction, respectively.

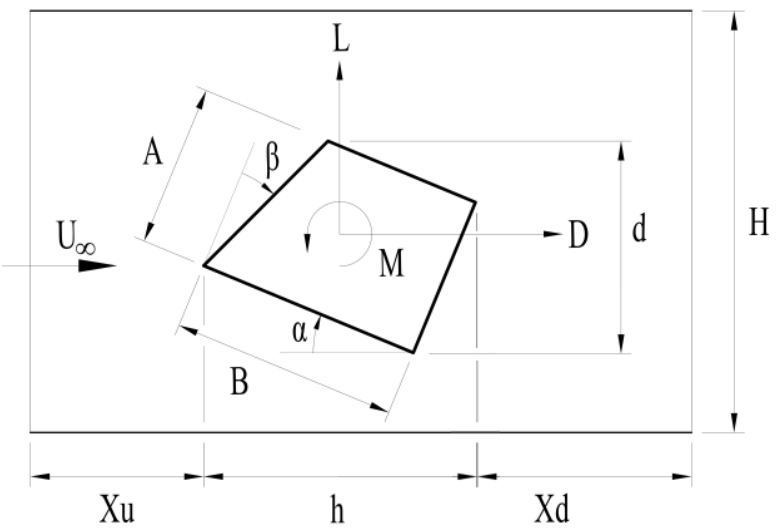

Fig. 3. Schematic of the computational geometry

\section{Numerical details}

The following numerical parameter were used in this study, unless otherwise specific, $\operatorname{Re}=100, \alpha=0^{\circ}, \beta=$ $22.5^{\circ}, B / A=1, \gamma=0.05, X_{u}=10$ and $X_{d}=15, \zeta=0.004$ and extends all side of 5 units from cylinder, the mesh distribution was made non-uniform with a vary mesh size of 0.004-0.5 and outside a region from the cylinder which extends 5 units, the mesh distribution was made uniform with a constant mesh size $\Delta=0.5$, the number of nodes on each side of cylinder $N_{b}=20$ nodes as shown in Fig. 4.

A finite volume method, non-uniform meshing with second-order implicit time discretization into eightnode quadratic quadrilateral elements is employed. An incompressible flow SIMPLEC code with constant fluid properties such as kinematic viscosity and density are used and the convective terms using a third-order QUICK scheme same as [1-3]. At the inlet which is located $X_{u}$ units upstream from the cylinder, a uniform flow velocity was definded in streamwise direction $U=$ 1 , in tranverse streamwise direction $V=0$. At the outlet which is located $X_{d}$ units downstream from the cylinder,

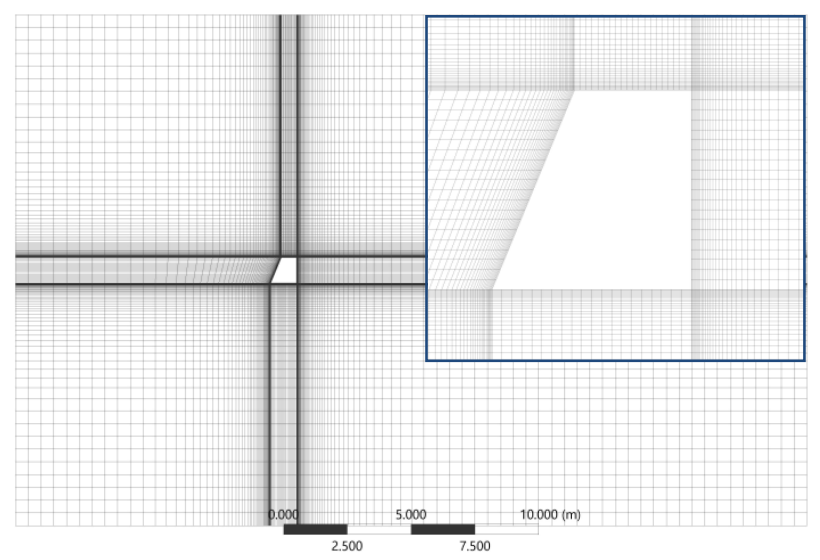

Fig. 4. Computational mesh for $\alpha=0^{\circ}$ and $\beta=22.5^{\circ}$, on the right top is near cylinder the zero gage pressure is definded. The confined flow surface frictionless stationary wall with specific shear stress $=0(V=0)$ at upper and lower side of cylinder $(H / 2)$ are employed and the right trapezoidal cylinder surface is prescribed to No-slip stationary wall condition $(U=0, V=0)$ with double precision. Initiated calculations with the fluid at rest $(U=0, V=0)$ after that in few time step the flow velocity is increased smoothly to unity with constant $\Delta t=0.05$, maximum iteration per time step of 40 , the monitor convergence criteria in continuity and velocity are set to 0.001 to use compares with residual at the end of each iteration during iterative sequence.

\section{Result and discussion}

To avoid inaccuracy from numerical simulation, the study effect of modeling parameter are performed. By using the Strouhal number and RMS lift coefficients as indicator the strong sensitivity [2-3] represent to another sensitivity numerical parameters from fully saturated flow calculation.

\subsection{Influence of time step}

In order to capture the shedding phase and amplitude accuracy, the influence of the time step is investigated as presented in Table 1. These results indicated that with the decrease of time step the Strouhal number and RMS lift coefficient are increased. Decreasing it from 0.2 to 0.1 , the Strouhal number and RMS lift coefficient are increased of $3.03 \%$ and $2.29 \%$ respectively. Decreasing it further to 0.05 , the Strouhal number and RMS lift coefficient are increased of $0.76 \%, 0.87 \%$, respectively. It negligible changed and next decreasing to 0.025 , the Strouhal number and RMS lift coefficient are negligible changed. The time step of 0.05 and Courant number of 12.5 is chosen to used for grid independence study further from reason as mention above and minimize the Courant number. This value less than [1-3] which them used 0.025 .

Table 1. Effect of time step.

\begin{tabular}{|c|c|c|c|}
\hline $\begin{array}{c}\text { Courant } \\
\text { number }\end{array}$ & $\boldsymbol{\Delta t}$ & $\boldsymbol{S t}$ & $\boldsymbol{C l}$ \\
\hline 50.0000 & 0.2000 & 0.1471 & 0.7405 \\
\hline 25.0000 & 0.1000 & 0.1515 & 0.7575 \\
\hline 12.5000 & 0.0500 & 0.1527 & 0.7641 \\
\hline 6.2500 & 0.0250 & 0.1533 & 0.7683 \\
\hline
\end{tabular}

\subsection{Grid independence study}

To avoid rapidly changing in high gradient area [1] the grid distribution is studied, the result is shown in Table 2. Decreasing the size domain next to cylinder from 0.004 to 0.002 , consequently grid density changed $150 \times 138$ to $151 \times 139$. From the result both the Strouhal number and RMS lift coefficient are decreased of $0.76 \%$ and $0.24 \%$ respectively. More additional study, fixed the size domain next to cylinder of 0.002 , increase the nodes on each side of cylinder to 40 nodes per cylinder side, 
the Strouhal number and RMS lift coefficient are again increased of $0.76 \%$ and $0.12 \%$ respectively. Finally it is decided to used the size domain next to cylinder by 0.004 , grid density $150 \times 138$ and the nodes on each side of cylinder to 20 nodes for further investigate.

Table 2. Effect of grid at $\Delta t=0.05$.

\begin{tabular}{|c|l|c|c|c|}
\hline $\boldsymbol{\zeta}$ & $\boldsymbol{N b}$ & Grid & $\boldsymbol{S t}$ & $\boldsymbol{C l}$ \\
\hline 0.004 & 20 & $150 \times 138$ & 0.1527 & 0.7641 \\
\hline 0.002 & 20 & $151 \times 139$ & 0.1515 & 0.7622 \\
\hline 0.002 & 40 & $171 \times 159$ & 0.1527 & 0.7631 \\
\hline
\end{tabular}

\subsection{Influence of domain size, upstream extents, downstream extents, convergence criteria and blockage ratio}

To study influence of upstream and downstream extents distance on present flow past cylinder. From previous decided, increase $X_{u}$ to 15 units, increase $X_{d}$ to 20 units and reduced $\gamma$ to $2.5 \%$ result as presented in Table 3 . The results indicated that with the increase $X_{u}$ from 10 to 15 unit the Strouhal numer and RMS lift coefficient are negligible decreased less than $1 \%$ and with the increase $X_{d}$ from 15 to 20 unit, the Strouhal number not changed and RMS lift coefficient is slightly increased. Where reduced $\gamma$ to $2.5 \%$ the result indicated that both the Strouhal number and RMS lift coefficient are slightly decrease of $0.76 \%$ and $1.24 \%$, respectively. In contrast, refined convergence criteria to 0.0001 , both parameter are negligible increased. It finally was decided that increasing $X_{u}, X_{d}$, domain size and convergence criteria not effected to previous decided. So it reasonable to final concluded numerical parameter to utility on this and further study of typical cylinder are following; $\gamma=0.05$, $X_{u}=10$ unit, $X_{d}=15$ units, $\zeta=0.004, \zeta-\Delta=0.004-0.5, \Delta$ $=0.5, N_{b}=20$ nodes almost aligned with [1-3]

Table 3. Effect of domain size at Courant number $=12.5, \Delta t=$ $0.05, \zeta=0.004, N b=20$ and Grid $=150 \times 138$.

\begin{tabular}{|c|c|c|c|c|c|}
\hline $\boldsymbol{X}_{\boldsymbol{u}}$ & $\boldsymbol{X}_{\boldsymbol{d}}$ & $\begin{array}{c}\text { Convergence } \\
\text { criteria }\end{array}$ & $\boldsymbol{\gamma}$ & $\boldsymbol{S t}$ & $\boldsymbol{C l}$ \\
\hline 10 & 15 & 0.001 & $5.0 \%$ & 0.1527 & 0.7641 \\
\hline 15 & 15 & 0.001 & $5.0 \%$ & 0.1515 & 0.7566 \\
\hline 10 & 20 & 0.001 & $5.0 \%$ & 0.1527 & 0.7646 \\
\hline 10 & 15 & 0.001 & $2.5 \%$ & 0.1515 & 0.7546 \\
\hline 10 & 15 & 0.0001 & $5.0 \%$ & 0.1538 & 0.7701 \\
\hline
\end{tabular}

\subsection{Effect of sharpening angle}

Fig. 5. Shows the time histories of the RMS lift coefficient of $\beta=0^{\circ}, 5^{\circ}, 22.5^{\circ}$ and $45^{\circ}$. It is indicated that for $\beta=0$, which is square cylinder, symmetry. Where sharpening angle is increased, RMS lift coefficient evidently increase but the Strouhal number constant that conformable to square cylinder at some approaching angle [4]. From Table 4. It can see that flow past the right trapezoidal cylinder is quite similar to the square cylinder on phase of transverse oscillation although in RMS lift coefficient is more effect than square cylinder at zero flow approaching but tend to similar to the square cylinder with flow approaching rather than zero.

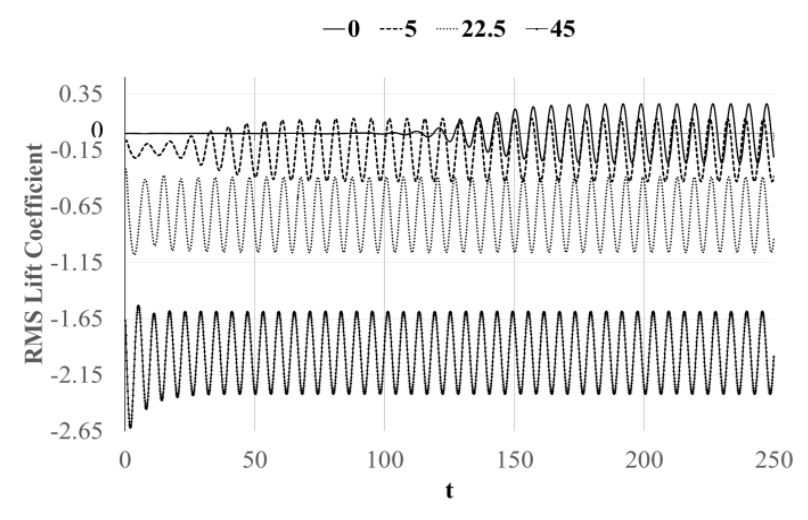

Fig. 5. Effect of sharpening angle on RMS lift coefficient

Table 4. Comparison $S t$ and $C l$ ' at $R e=100$ with other cylinder cross-section.

\begin{tabular}{|l|l|l|l|l|}
\hline $\begin{array}{c}\text { Approaching } \\
\text { angle }\end{array}$ & $\begin{array}{c}\text { Type of } \\
\text { cylinder }\end{array}$ & \multicolumn{1}{|c|}{$\boldsymbol{\gamma}$} & \multicolumn{1}{|c|}{$\boldsymbol{S t}$} & $\boldsymbol{C l}$ \\
\hline $\boldsymbol{\alpha}=0^{\circ}$ & Square [3] & 0.05 & 0.1460 & 0.1560 \\
\hline & Square [4] & 0.025 & 0.1447 & 0.1908 \\
\hline & Present & 0.05 & 0.1527 & 0.7641 \\
\hline $\boldsymbol{\alpha}=22.5^{\circ}$ & Square [4] & 0.025 & 0.1408 & 0.5437 \\
\hline $\boldsymbol{\alpha}=45^{\circ}$ & Square [4] & 0.025 & 0.1388 & 0.6539 \\
\hline
\end{tabular}

\section{Conclusions}

Numerical simulation of two-dimensional laminar flow past a right trapezoidal cylinder in unsteady flow at $R e=$ $100, \alpha=0^{\circ}$ and $\beta=22.5^{\circ}$ with $B / A=1$ have been carried out. The flow past the right trapezoidal cylinder is slightly difference to the square cylinder on phase of transverse oscillation at zero flow approaching, The RMS lift coefficient is increased follow sharpening angle increasing and to seem to similar square cylinder at flow approaching rather than zero.

The increasing of sharpening angle, the Strouhal number is negligible changed whilst the RMS lift coefficients significantly increased.

The Strouhal number of 0.1527 and RMS lift coefficient of 0.7641 in this study are provided for more applicable data for engineering design of barred tee in aspect of structural integrity further.

\section{References}

1. A. Sohankar, L. Davison, C. Norberg, Proc. Twelfth Australasian Fluid Mech. Conf., 517-520 (1995)

2. A. Sohankar, C. Norberg, L. Davison, J. Wind Eng. Ind. Aerodyn 69-71, 189-201 (1997)

3. A. Sohankar, C. Norberg, L. Davison, Int. J. Numer. Meth. Fluids 26, 39-56 (1998)

4. M. Zhao, L. Cheng, T. Zhou, Phys. Fluids 25, 023603-25 (2013)

5. A. Kumar, A. Dalal, Int. J. Numer. Meth. Fluids 52, 801-821 (2006)

6. T. Prasanth, S. Behara, S. Singh, R. Kumar, S. Mittal, J. Fluids and Structures 22, 865-876 (2006) 